\title{
Effects of carbon dioxide purities on mitotic index in lymphocyte culture and metaphase chromosome quality
}

\author{
SOFIATI PURNAMI ${ }^{1, \bullet}$, INDRI PERMATA WIBISARI ${ }^{2}$, VIRIA AGESTI SUVIFAN ${ }^{1}$, SITI NURHAYATI $^{1}$, \\ DWI RAMADHANI ${ }^{1,3, \bullet \bullet}$ \\ ${ }^{1}$ Centre for Technology of Radiation Safety and Metrology, National Nuclear Energy Agency. Jl. Lebak Bulus Raya No. 49, Jakarta Selatan 12440, \\ Jakarta, Indonesia. `email: ipungsp@batan.go.id. \\ ${ }^{2}$ Department of Biology, Faculty of Mathematics and Natural Sciences, Universitas Negeri Jakarta. Jl. Rawamangun Muka, Rawamangun, Jakarta Timur \\ 13220, Jakarta, Indonesia. \\ ${ }^{3}$ Doctoral Program in Biomedical Sciences, Faculty of Medicine, Universitas Indonesia. Jl. Salemba Raya No. 6, Ged. IMERI, Tower b, Lt. 11, Jakarta \\ Pusat 10430, Jakarta, Indonesia.vvemail: dhani02@batan.go.id
}

Manuscript received: 21 July 2021. Revision accepted: 29 September 2021.

\begin{abstract}
Purnami S, Wibisari IP, Suvifan VA, Nurhayati S, Ramadhani D. 2021. Effects of carbon dioxide purities on mitotic index in lymphocyte culture and metaphase chromosome quality. Nusantara Bioscience 13: 171-176. The metaphase chromosome spread quality is necessary for a faster individual dose prediction following radiological accidents using dicentric chromosome assay. It is well known that the low-quality metaphase chromosome spreads can lead to false positives of dicentric chromosome identification. Thus, evaluating the main variable that influences the preparation of high-quality metaphase chromosome spread is important. Until now, no studies have assessed the effects of $\mathrm{CO}_{2}$ purities on metaphase chromosome spread quality. This study aimed to evaluate the effects of carbon dioxide $\left(\mathrm{CO}_{2}\right)$ purities on lymphocyte proliferation and the quality of metaphase chromosome spreads to improve the chromosome aberration assay for cytogenetic biodosimetry purposes. Whole blood samples from three subjects were cultured and incubated for 48 hours with two different grades of $\mathrm{CO}_{2}$ (high purity and food grades) and without $\mathrm{CO}_{2}$. Each subject's mitotic index (MI) was assessed, and the quality of metaphase chromosome spreads was evaluated by comparing the lengths of chromosomes 1,2 , and 21. Statistical analysis revealed that the difference between manual and automatic MI under three conditions of $\mathrm{CO}_{2}$ purity was not statistically significant $(p$ $=0.162 ; p=0.901)$. Comparative analysis of the lengths of chromosomes 1, 2, and 21 from 145 metaphases also showed a difference that was not statistically significant $(p=0.745 ; p=0.915 ; p=0.399)$. Our findings suggest that $\mathrm{CO}_{2}$ purities do not impair lymphocyte proliferation or metaphase quality. Further investigation should include other technical improvements, such as drop-slide optimization.
\end{abstract}

Keywords: $\mathrm{CO}_{2}$, lymphocytes, metaphase, Mitotic Index, purity

\section{INTRODUCTION}

Radiological accidents that cause unregulated radiation exposure to humans can occur anytime in human life. Commonly, victims in these situations are not carrying a personal dosimeter. The only way to estimate the individual dose of radiation in such cases is through biological dosimetry, a method that offers the possibility of separate dose estimation weeks or even months after the exposure event (Ivashkevich et al. 2017; Kulka et al. 2018). Biological dosimetry (biodosimetry) is the process of estimating the radiation dose received by an individual during accidental exposure using a biological marker (Perumal et al. 2015; Herate and Sabatier, 2020). Among many methods of the biological assay in biodosimetry, scoring of the dicentric chromosomes (DC) in peripheral blood lymphocytes (PBL) is considered a reliable and sensitive method due to the specificity for ionizing radiation and the low background frequency of DC in PBL (Mayakannan et al. 2018; Han et al. 2020; Pujol-Canadell et al. 2020). Dicentric chromosomes, defined as chromosomes with two centromeres, are formed from the fusion of two chromosome segments (Figure 1). The DC assay (DCA) is commonly performed after 48 hours of whole blood culture with phytohemagglutinin (PHA), followed by 2 hours of incubation with a mitosis-blocking agent colcemid or colchicine. The harvest process, consisting of hypotonic treatment and fixation, is performed after culture. Slide preparation and staining of the metaphase chromosomes with Giemsa follow the harvest process (Han et al. 2020; Xiao et al. 2020). Unstable chromosome aberrations (CAs) such as DC, ring chromosomes (R), and acentric fragment (AF) chromosomes can be scored for dose estimation within a few days after irradiation.

It is well known that chromosome morphology is highly variable between metaphase cells ( $\mathrm{Li}$ et al. 2016). Therefore, low-quality chromosome spreads due to highly condensed chromosomes, overlapping chromosomes and cytoplasmic background, or overlapping non-stimulated lymphocytes can lead to false positives of dicentric chromosome identification. In addition, low-quality chromosome spreads may delay individual dose predictions using DC markers. 


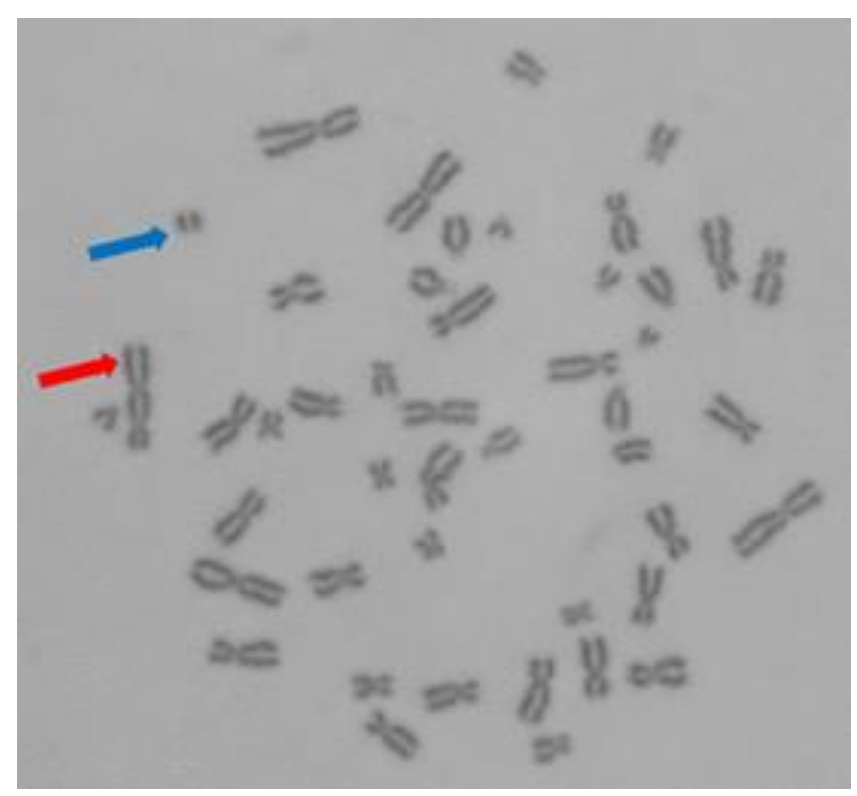

Figure 1. Dicentric chromosome (red arrow) and accompanying acentric fragment (blue arrow) (International Atomic Energy Agency 2011)

The DCA, the protocol standardized by the International Organization for Standardization, is highly sensitive, and the background frequency of DC in humans is low (1-2 per 1000 metaphases) (Wilkins et al. 2011). In contrast, higher-quality chromosome spreads and higher numbers of metaphase cells could increase the speed of individual dose prediction following radiological accidents. Until now, numberless chromosome preparations with the primary goal of producing high-quality metaphase spreads for chromosome analysis have been made by cytogeneticists. However, variation in the metaphase extends the quality produced in the same laboratory (Claussen et al. 2002). The inconsistency of metaphase spread quality could affect the cytogenetic results and often contribute to difficulty in chromosome analysis (Ami et al. 2014). Numerous studies have already been performed to increase chromosome spreading, which is still not completely understood until now. Henegariu et al. (2001) used a water bath and a metal plate to control the slide temperature to customize the chromosome's spread quality in G-banding or fluorescence in situ hybridization (FISH) techniques. Another study by Deng et al. (2003) used a water bath to provide moisture and a suitable temperature for chromosome spreading and cell drying. Kwasny et al. (2014) even developed a microfluidic device based on Cyclic Olefin Copolymer (COC) material for reliable chromosome spreading for G-banding and FISH techniques.

The quality of metaphase spread determines fixative evaporation in a slide during chromosome preparation. Climatic conditions, such as relative humidity and room temperature, influence this process. In addition, other variables such as the distance from the fixed mitotic cells are dropped onto the slide, the diameter of the opening of the pipette, which determines the size of the drops, the amount of evaporating fixative on the slide, and the slide temperature during evaporation of the fixative are also considered to influence the preparation of high-quality metaphase spreads. However, to our knowledge, no previous studies have assessed the effects of $\mathrm{CO}_{2}$ purities on MI and metaphase chromosome spread quality. Therefore, this study aimed to understand the impact of carbon dioxide $\left(\mathrm{CO}_{2}\right)$ purities on a mitotic index (MI) in lymphocyte culture and metaphase chromosome spread quality. The length of the two most extended chromosomes, chromosomes 1 and 2, and the shortest chromosome, chromosome 21 , were used as metaphase chromosome spread quality parameters. Chromosomes 1 and 2 are considered the most extended chromosomes, whereas chromosome 21 is the shortest in the human genome (Morton 1991; Piovesan et al. 2019).

\section{MATERIALS AND METHODS}

\section{Blood cultures}

With informed consent, whole blood samples were collected from three healthy female donors of similar age (21 years). These three female donors have different blood types. The first donor has a B type, and the second donor has an $\mathrm{O}$ type, whereas the last donor has an $\mathrm{AB}$ type. Blood cultures were then performed by mixing the blood samples with $7.5 \mathrm{~mL}$ Roswell Park Memorial Institute (RPMI) 1640, $1 \mathrm{~mL}$ Fetal Bovine Serum (FBS), $0.1 \mathrm{~mL}$ streptomycin/penicillin, and $0.12 \mathrm{~mL}$ phytohemagglutinin (PHA). Cultures were incubated for $48 \mathrm{hr}$ at $37{ }^{\circ} \mathrm{C}$ in $5 \%$ $\mathrm{CO}_{2}$ (Ramadhani et al. 2018). Two different $\mathrm{CO}_{2}$ purities were applied during incubation: high purity (99.99\%) and food-grade purity $(99.90 \%)$. Incubation without the presence of $\mathrm{CO}_{2}$ was also performed. At $45 \mathrm{hr}$ of incubation, colchicine $(0.05 \mu \mathrm{g} / \mathrm{mL}$ final concentration $)$ was added to the cultures. The harvest process was then performed for each culture by centrifuging the culture flask for $10 \mathrm{~min}$ at $214 \times \mathrm{g}$, removing the supernatant, and resuspending the pellet in $7 \mathrm{~mL}$ pre-warmed $\left(37^{\circ} \mathrm{C}\right) 0.075$ $\mathrm{M} \mathrm{KCl}$. Suspensions were incubated for 20 minutes in a 37 ${ }^{\circ} \mathrm{C}$ water bath and centrifuged for 10 minutes at $214 \times \mathrm{g}$. Cells underwent two rounds of fixation using $7 \mathrm{~mL}$ fixative solution (3:1 methanol: acetic acid) and were stored at $4{ }^{\circ} \mathrm{C}$ overnight. The fixed cells were then spread onto clean, wet mount slides, dried, and stained with $4 \%$ Giemsa solution ( $\mathrm{pH}=6.8$ ) for 10 minutes.

\section{Mitotic Index calculation}

Mitotic Index calculation was performed manually and automatically using the protocol described in our previous publication (Ramadhani et al. 2018). The manual analysis of MI was conducted based on a protocol published by the International Atomic Energy Agency (2011). Specifically, the number of metaphase cells per 1,000 total metaphase and blast cells was counted at $400 \times$ magnification using a light microscope. The number of metaphase cells was then converted to a percentage to calculate MI. The automatic calculation of MI was performed using the Metafer 3.11.2 imaging system (MetaSystems, Altlussheim, Germany), by 
which the entire area of the slide was scanned using the metaphase finder (MSearch) module from the Metafer 3.11.2 connected to a Zeiss Axioplan 2 Imaging epifluorescent microscope equipped with a Cool Cube (MetaSystems, Altlussheim, Germany).

\section{Chromosomes 1, 2, and 21 length measurements}

This study's metaphase chromosome spread quality was interpreted as the length of chromosomes 1, 2, and 21. This method argues that longer chromosome length results in easier dicentric identification and, thus, a faster individual dose prediction. Metaphase cell images were acquired using a Zeiss Axioplan 2 Imaging epifluorescence microscope connected to a Cool Cube digital highresolution CCD camera using Metafer software, 3.11.2 (MetaSystems, Altlussheim, Germany). Images were exported as .tif files with a resolution of $1280 \times 1024$ pixels. A total of 135 images were used in this study. The lengths of chromosomes 1, 2, and 21 were measured using the ImageJ program. Before a length measurement is performed in this program, a pixel calibration must be conducted using the ImageJ "Set scale" command and a known distance from an image captured using the same imaging system and configuration. Fluorescent beads with a known diameter of $2.5-\mathrm{mm}$ were used in this study to perform pixel calibration, and 12 pixels in our images were determined to represent $1 \mathrm{~mm}$.

\section{Statistical analysis}

The Shapiro-Wilk test was used to assess the normality of the distribution of the data. In addition, a One-Way ANOVA test was used to evaluate the differences in MI and lengths of chromosomes 1, 2, and 21 between all groups. Statistical analysis was performed with Minitab
(Minitab Inc., USA) version 19. Significance was considered as $p<0.05$.

\section{RESULTS AND DISCUSSION}

The effects of two different $\mathrm{CO}_{2}$ purities on $\mathrm{MI}$ and chromosome spread quality were investigated in this study. The means of manual and automatic MI calculations for each $\mathrm{CO}_{2}$ group were not significantly different ( $p=$ $0.162 ; p=0.901$ ) (Table 1). However, a higher MI value was observed with high-purity $\mathrm{CO}_{2}$ (Figure 2).

$\mathrm{CO}_{2}$ purity had no significant effect on the lengths of chromosomes 1, 2, and 21 (Table 2, Figure 3). Statistical analysis also revealed no significant difference ( $p=$ $0.745 ; p=0.915 ; p=0.399)$.

It is well known that high-quality chromosome spreads are essential in cytogenetic analysis, including biodosimetry (Howe et al. 2014; Yao et al. 2020). A wellspread chromosome will result in a suitable chromosome morphology for identifying DC. Furthermore, in the case of mass-casualty nuclear accidents involving many individuals, automatic dicentric scoring might provide a fast and reliable tool for individual dose prediction (Romm et al. 2013). However, the quality of metaphase images determines the success of automatic dicentric scoring. Moreover, many conditions could affect the spreading chromosome rate, such as humidity, temperature, the object-glass slide quality, and temperature while dropping the fixed cells. Therefore, obtaining the excellent quality spreads of metaphase chromosomes is often regarded as an art instead of a science in cytogenetic analysis (Kwasny et al. 2014).

Table 1. Mean manual and automatic MI values of three different $\mathrm{CO}_{2}$ groups

\begin{tabular}{ccccccc}
\hline \multirow{2}{*}{ Donor } & \multicolumn{3}{c}{ Mean of manual MI value \pm SD } & \multicolumn{2}{c}{ Mean of automatic MI value \pm SD } \\
\cline { 2 - 7 } & $\begin{array}{c}\text { High purity } \\
\mathbf{C O}_{\mathbf{2}}\end{array}$ & $\begin{array}{c}\text { Food grade } \\
\mathbf{C O}_{\mathbf{2}}\end{array}$ & $\begin{array}{c}\text { Without continuous } \\
\mathbf{C O}_{\mathbf{2}}\end{array}$ & $\begin{array}{c}\text { High purity } \\
\mathbf{C O}_{\mathbf{2}}\end{array}$ & $\begin{array}{c}\text { Food grade } \\
\mathbf{C O}_{\mathbf{2}}\end{array}$ & $\begin{array}{c}\text { Without continuous } \\
\mathbf{C O}_{\mathbf{2}}\end{array}$ \\
\hline 1 & $8.40 \pm 2.69$ & $7.33 \pm 1.20$ & $5.67 \pm 0.57$ & $17.07 \pm 0.368$ & $20.68 \pm 1.96$ & $19.71 \pm 2.56$ \\
2 & $9.80 \pm 2.62$ & $6.80 \pm 1.05$ & $7.80 \pm 2.09$ & $17.49 \pm 1.242$ & $14.58 \pm 3.95$ & $12.65 \pm 0.906$ \\
3 & $13.80 \pm 1.38$ & $11.73 \pm 1.86$ & $11.13 \pm 0.70$ & $39.52 \pm 3.58$ & $34.48 \pm 0.83$ & $35.22 \pm 1.369$ \\
Mean & $10.67 \pm 2.80$ & $8.62 \pm 2.71$ & $8.20 \pm 2.75$ & $24.69 \pm 12.84$ & $23.25 \pm 10.20$ & $22.53 \pm 11.55$ \\
\hline
\end{tabular}
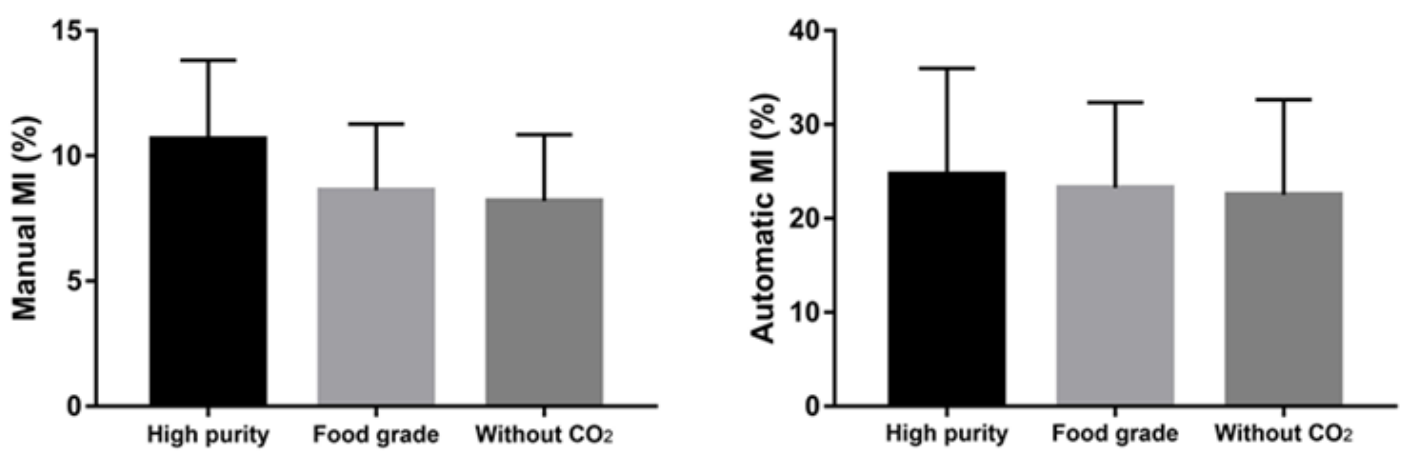

Figure 2. Bar graph of mean manual and automatic MI values in all $\mathrm{CO}_{2}$ groups 
Table 2. Mean length of chromosomes 1,2 , and 21 of three different $\mathrm{CO}_{2}$ groups

\begin{tabular}{cccccccccc}
\hline & \multicolumn{2}{c}{ Chromosome 1 length $(\boldsymbol{\mu m})$} & \multicolumn{2}{c}{ Chromosome 2 length $(\boldsymbol{\mu m})$} & \multicolumn{2}{c}{ Chromosome 21 length $(\boldsymbol{\mu m})$} \\
\cline { 2 - 10 } Donor & $\begin{array}{c}\text { High purity Food-grade } \\
\mathbf{C O}_{\mathbf{2}}\end{array}$ & $\begin{array}{c}\text { Without } \\
\text { continuous } \\
\mathbf{C O}_{\mathbf{2}}\end{array}$ & $\begin{array}{c}\text { High purity } \\
\mathbf{C O}_{\mathbf{2}}\end{array}$ & $\begin{array}{c}\text { Food-grade } \\
\mathbf{C O}_{\mathbf{2}}\end{array}$ & $\begin{array}{c}\text { Without } \\
\text { continuous } \\
\mathbf{C O}_{\mathbf{2}}\end{array}$ & $\begin{array}{c}\text { High purity } \\
\mathbf{C O}_{\mathbf{2}}\end{array}$ & $\begin{array}{c}\text { Food-grade } \\
\mathbf{C O}_{\mathbf{2}}\end{array}$ & $\begin{array}{c}\text { Without } \\
\text { continuous } \\
\mathbf{C O}_{\mathbf{2}}\end{array}$ \\
\hline 1 & $4.007 \pm 0.480$ & $4.648 \pm 0.411$ & $4.462 \pm 0.322$ & $3.788 \pm 0.365$ & $4.405 \pm 0.491$ & $4.303 \pm 0.270$ & $1.072 \pm 0.038$ & $1.181 \pm 0.029$ & $1.164 \pm 0.103$ \\
2 & $4.484 \pm 0.060$ & $4.543 \pm 0.191$ & $4.624 \pm 0.206$ & $4.376 \pm 0.081$ & $4.338 \pm 0.212$ & $4.253 \pm 0.245$ & $1.171 \pm 0.047$ & $1.227 \pm 0.116$ & $1.341 \pm 0.213$ \\
3 & $4.385 \pm 0.316$ & $4.119 \pm 0.234$ & $4.096 \pm 0.777$ & $4.316 \pm 0.385$ & $3.912 \pm 0.152$ & $3.875 \pm 0.680$ & $1.177 \pm 0.073$ & $1.132 \pm 0.037$ & $1.127 \pm 0.061$ \\
Mean & $4.292 \pm 0.362$ & $4.436 \pm 0.388$ & $4.394 \pm 0.070$ & $4.159 \pm 0.388$ & $4.218 \pm 0.361$ & $4.143 \pm 0.435$ & $1.140 \pm 0.070$ & $1.180 \pm 0.075$ & $1.210 \pm 0.157$ \\
\hline
\end{tabular}

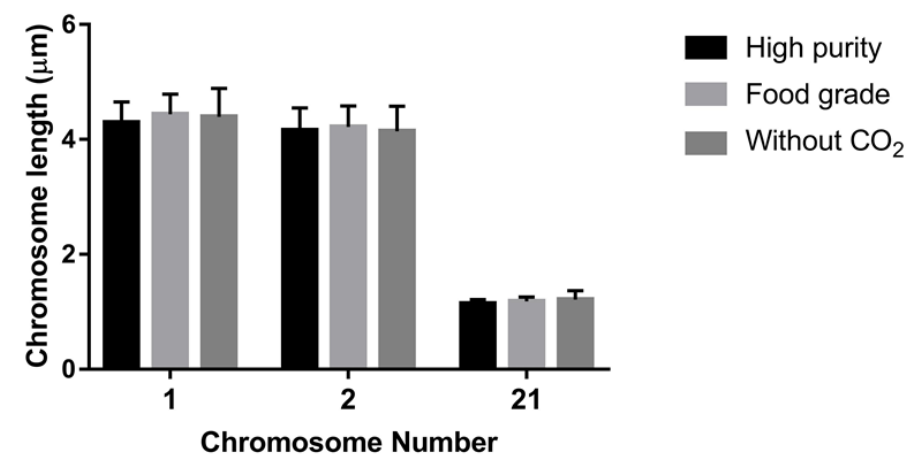

Figure 3. Bar graph showing the mean length of chromosomes 1, 2, and 21 in all donors

Currently, an alternative scoring method termed DCA QuickScan has been established to obtain much faster radiation dose information with acceptable accuracy. In the DCA QuickScan triage approach, the number of metaphase spreads that should be examined is much lower (only 50) than in conventional DCA, which requires 500 or 1,000 cells to be evaluated (ICRU 2019). Furthermore, the number of all chromosomal damages, including DC, R, and $\mathrm{AF}$, was recorded without considering each cell's chromosome number. Thus, in the DCA QuickScan triage approach, the quality of metaphase spread becomes essential to ensure the fast process of radiation dose prediction. Therefore, metaphase spread quality is critical for highly accurate DC identification (Liu et al. 2017).

This study aimed to assess whether different $\mathrm{CO}_{2}$ purities could enhance lymphocyte proliferation and metaphase quality. It is also established that $\mathrm{CO}_{2}$ levels during incubation are an essential parameter for culture and can influence the $\mathrm{pH}$ of the culture medium. Blood cultures should experience optimal temperature, humidity, and $\mathrm{pH}$ conditions until sufficient numbers of dividing cells are present. Blood cultures for cytogenetic analysis can be maintained in "open" or "closed" systems. Open systems allow for the free exchange of gases between the insides of culture flasks and the surrounding environment inside the incubator. Culture flask caps are only loosely applied in these systems to facilitate gas exchange. Open systems require a $\mathrm{CO}_{2}$ incubator to maintain the $5 \% \mathrm{CO}_{2}$ level necessary for sustaining the ideal $\mathrm{pH}$ of 7.2-7.4. A major drawback to these systems is the susceptibility to microbial contamination, particularly fungi, due to the moist, warm conditions inside the incubator (Keagle and Gersen 2013).
In contrast to open systems, culture flasks in closed systems must be tightly capped to prevent gas exchange. Therefore, $\mathrm{CO}_{2}$ incubators are not necessarily required in closed systems (O'Brien et al. 2020). In short-term cultures such as blood culture, the commercial media is expected to be buffered to the appropriate $\mathrm{pH}$. Furthermore, microbial contamination does not pose a significant risk in closed systems (Keagle and Gersen 2013). In our study, we decided to use an open system for cultures in the $\mathrm{CO}_{2}$ incubator since we aimed to investigate the effects of $\mathrm{CO}_{2}$ purities on $\mathrm{MI}$ and chromosome spread quality.

The study revealed that $\mathrm{CO}_{2}$ purity did not affect $\mathrm{MI}$ or chromosome spread quality (Table 1). A small percentage of $\mathrm{CO}_{2}$ impurity evidence did not impair cell proliferation or chromosome spread quality. The American Type Culture Collection (ATCC) recommends medical-grade $\mathrm{CO}_{2}$ in cell culture incubators. However, this study's findings suggest that food-grade $\mathrm{CO}_{2}$ is sufficient for use in cell cultures. Another advantage to using food-grade $\mathrm{CO}_{2}$ is the lower cost compared to high-purity $\mathrm{CO}_{2}$. In this study, chromosome length was used to interpret good chromosome spread quality since it is easier to identify chromosome aberrations, particularly dicentric chromosomes, in longer chromosomes (Kato 2019). However, using only the chromosome length as a parameter for determining chromosome spread quality may not be sufficient. Evaluation of other characteristics, such as metaphase area, metaphase chromosome number, metaphase chromosome overlapping number, and intact metaphase number, as performed by Deng et al. (2003), might be more comprehensive. Additional assessment of systematically scoring chromosome spread quality could also be conducted in further investigation. For example, 
calculating the percentage of metaphase with non-separated chromatids or twisted chromosomes and metaphase with non-visible centromeric constrictions may be a valuable additional assessment. According to Gruel et al. (2013) manually excluded these types of metaphase, together with metaphases containing a chromosome number other than 46 and metaphases in second or third cell division, to increase the success of automatic dicentric identification using DCScore software from Metasystems. Based on this study, the lengths of chromosomes 1 and 2 ranged from 4.143 to $4.436 \mathrm{~mm}$ (Table 2). These values are comparable to those found in Koyani and Saiyad's (2011) survey, where the most extended chromosomes ranged from 3.80 to $4.90 \mathrm{~mm}$ and were found in blood cultures exposed to colchicine for 4 hours. In contrast, in our study, the colchicine exposure time was 3 hours.

The manual MI values of all donors in this study are also comparable with a previous study by Viswanathan et al. (2019) that found the percentage of MI in different blood types varied widely. The rate of MI in variety $\mathrm{A}$ ranged from 4.39 to $18.6 \%$, with mean \pm SD values of 11.0 $\pm 4.3 \%$. In type $\mathrm{B}$, the percentage of $\mathrm{MI}$ ranged from 4.7 to $23.1 \%$, with mean \pm SD values of $12.19 \pm 4.4 \%$. Then, the rate of $\mathrm{MI}$ in the $\mathrm{AB}$-type ranged from 8.5 to $24.6 \%$, with mean $\pm \mathrm{SD}$ values of $12.5 \pm 5.3 \%$. Last, the percentage of $\mathrm{MI}$ in $\mathrm{O}$ type ranged from 4.1 to $24.6 \%$, with mean \pm SD values of $12.2 \pm 4.9$. This current study showed that the mean manual $\mathrm{MI}$ values range in all $\mathrm{CO} 2$ groups of donor 1 , which had the B type, were 5.67 to $8.40 \%$. In donor $2(\mathrm{O}$ type), the mean manual $\mathrm{MI}$ values range in all $\mathrm{CO}_{2}$ groups started from 6.80 to $9.80 \%$. Donor 3 with AB type had the mean manual $\mathrm{MI}$ values range in all $\mathrm{CO}_{2}$ groups from 11.13 to $13.80 \%$.

In conclusion, $\mathrm{CO}_{2}$ purities impaired neither lymphocyte proliferation nor metaphase chromosome quality. Even though the higher mean manual and automatic MI values were observed in the culture with high purity $\mathrm{CO}_{2}(10.67 \pm 2.80 \%$ and $24.69 \pm 12.84 \%)$, statistical analysis revealed non-significant results compared to food-grade $\mathrm{CO}_{2}$ and without continuous $\mathrm{CO}_{2}$. Statistical analysis also revealed a non-significant difference in chromosome lengths in all groups. The most extended chromosomes 1, 2, and 21 were observed in cultures with food-grade $\mathrm{CO}_{2}$, not in high-purity $\mathrm{CO}_{2}$ $(4.436 \pm 0.388$ and $4.218 \pm 0.361 \mathrm{~mm})$. Based on this study's findings, it is clear that other technical improvements, such as drop-slide optimization, should be conducted in future studies to obtain a high-quality chromosome spread.

\section{ACKNOWLEDGEMENTS}

The authors gratefully acknowledge the technical contributions of Mrs. Yanti Lusiyanti. We also thank Prof. Mukh Syaifudin for the valuable comments and suggestions, which improve the quality of the article.

\section{REFERENCES}

Ami D, Di Segni M, Forcella M et al. 2014. Role of water in chromosome spreading and swelling induced by acetic acid treatment: A FTIR spectroscopy study. Eur J Histochem 58(1): 33-39. DOI: 10.4081/ejh.2014.2330.

Claussen U, Michel S, Mühlig P et al. 2002. Demystifying chromosome preparation and the implications for the concept of chromosome condensation during mitosis. Cytogenet Genom Res 98(2-3): 136146. DOI: $10.1159 / 000069817$.

Deng W, Tsao SW, Lucas JN et al. 2003. A new method for improving metaphase chromosome spreading. Cytometry A51(1): 46-51. DOI: 10.1002/cyto.a.10004.

Gruel G, Grégoire E, Lecas S et al. 2013. Biological dosimetry by automated dicentric scoring in a simulated emergency. Radiat Res 179(5): 557-569. DOI: 10.1667/rr3196.1.

Han L, Yu G, Ping W et al. 2020. Cytogenetic biodosimetry for radiation accidents in China. Radiat Med Prot 1(3): 133-139. DOI: 10.1016/j.radmp.2020.09.001.

Henegariu O, Heerema NA, Lowe Wright L et al. 2001. Improvements in cytogenetic slide preparation: Controlled chromosome spreading, chemical aging and gradual denaturing. Cytometry 43(2): 101-109. DOI: CYTO1024>3.0.CO;2-8

Herate C, Sabatier L. 2020. Retrospective biodosimetry techniques: Focus on cytogenetics assays for individuals exposed to ionizing radiation. Mutat Res Rev 783: 108287. DOI: 10.1016/j.mrrev.2019.108287.

Howe B, Ayesha U, Fern T. 2014. Chromosome preparation from cultured cells. J Vis Exp 83: 3-7. DOI: 10.3791/50203.

ICRU. 2019. 3 Biodosimetry. J. ICRU 19(1): 26-45. DOI: 10.1177/1473669119893151.

International Atomic Energy Agency. 2011. Cytogenetic dosimetry: Applications in preparedness for and response to radiation emergencies. International Atomic Energy Agency, Vienna. http://www-pub.iaea.org/books/IAEABooks/8735/CytogeneticDosimetry-Applications-in-Preparedness-for-and-Response-toRadiation-Emergencies.

Ivashkevich AT, Ohnesorg C, Sparbier E et al. 2017. Advances in biological dosimetry. J Phys Conf Ser 777(012012): 1-6. DOI: 10.1088/1742-6596/755/1/011001.

Kato TA. 2019. Human lymphocyte metaphase chromosome preparation for radiation-induced chromosome aberration analysis. In: Clifton NJ (eds). Methods in Molecular Biology. Springer, New York. DOI: 10.1007/978-1-4939-9432-8_1.

Keagle MB, Steven LG. 2013. Basic cytogenetics laboratory procedures. In: Steven LG, Martha BK (eds). The Principles of Clinical Cytogenetics, Springer, New York. DOI: 10.1007/978-1-4419-1688$4 \_4$.

Koyani PR, Saiyad SS. 2011. Study of effect of colchicine exposure on length of chromosome during mitosis. J Anat Soc India 60: 177-180. DOI: 10.1016/S0003-2778(11)80020-1.

Kulka U, Wojcik A, Di Giorgio M et al. 2018. Biodosimetry and biodosimetry networks for managing radiation emergency. Radiat Prot Dosim 182: 128-138. DOI: 10.1093/RPD/NCY137.

Kwasny D, Olga M, Indumathi V et al. 2014. A semi-closed device for chromosome spreading for cytogenetic analysis. Micromachines 5: 158-170. DOI: $10.3390 / \mathrm{mi} 5020158$.

Li Y, Joan HK, Ruth CW et al. 2016. Automated discrimination of dicentric and monocentric chromosomes by machine learning-based image processing. Microsc Res Tech 79: 393-402. DOI: $10.1002 /$ jemt.22642.

Liu J, Li Y, Wilkins R et al. 2017. Accurate cytogenetic biodosimetry through automated dicentric chromosome curation and metaphase cell selection. F1000Research 6: 1396. DOI: 10.12688/f1000research.12226.1.

Mayakannan K, Sureka CS, Venkatesh R et al. 2018. Construction of dose response curves up to $6 \mathrm{~Gy}$ for micronucleus and dicentric chromosome aberration assay with $6 \mathrm{MV}$ X-Ray Beam. Radiat Meas 115: 60-68. DOI: 10.1016/j.radmeas.2018.05.012.

Morton NE. 1991. Parameters of the human genome. Proc Natl Acad Sci USA 88: 7474-7476. DOI: 10.1073/pnas.88.17.7474.

O'Brien MB, Rachel MM, Kieran GM. 2020. Application of the TruCulture ${ }^{\circledR}$ whole blood stimulation system for immune response profiling in cattle. Vet Immunol Immunopathol 221: 110025. DOI: 10.1016/j.vetimm.2020.110025. 
Perumal V, Tamizh SGS, Venkateswarlu R et al. 2015. Radiation signature on exposed cells: Relevance in dose estimation. World J Radiol 7: 266. DOI: 10.4329/wjr.v7.i9.266.

Piovesan A, Pelleri MC, Antonaros F et al. 2019. On the length, weigh and gc content of the human genome. BMC Res Notes 12: 106. DOI: 10.1186/s13104-019-4137-z.

Pujol-Canadell M, Jay RP, Lidia C et al. 2020. Cytogenetically-based biodosimetry after high doses of radiation. PLoS ONE 15: 1-12. DOI: 10.1371/journal.pone.0228350.

Ramadhani D, Nurhayati S, Rahardjo T et al. 2018. Lymphocyte proliferation kinetics in inhabitant of Takandeang Village, Mamuju: A high background radiation areas in Indonesia. Indones Biomed J 10(1): 66-73. DOI: 10.18585/inabj.v10i1.357.

Romm H, Ainsbury E, Barnard S et al. 2013. Automatic scoring of dicentric chromosomes as a tool in large scale radiation accidents.
Mutat Res Genet Toxicol Environ Mutagen 756: 174-183. DOI: 10.1016/j.mrgentox.2013.05.013.

Viswanathan S, Kanagaraj K, Raavi V et al. 2019. Does proliferation capacity of lymphocytes depend on human blood types? J Cell Biochem 120: 5722-5728. DOI: 10.1002/jcb.27858.

Wilkins RC, Romm H, Oestreicher U et al. 2011. Biological dosimetry by the triage dicentric chromosome assay - further validation of international networking. Radiat Meas 46: 923-928. DOI: 10.1016/j.radmeas.2011.03.012

Xiao C, Ningning H, Yang L et al. 2020. Research progress on biodosimeters of ionizing radiation damage. Radiat Med Prot 1: 127132. DOI: 10.1016/j.radmp.2020.06.002.

Yao Q, Gao J, Chen F et al. 2020. Development and application of an optimized drop-slide technique for metaphase chromosome spreads in maize. Biotech Histochem 95: 276-284. DOI: 10.1080/10520295.2019.1686167 